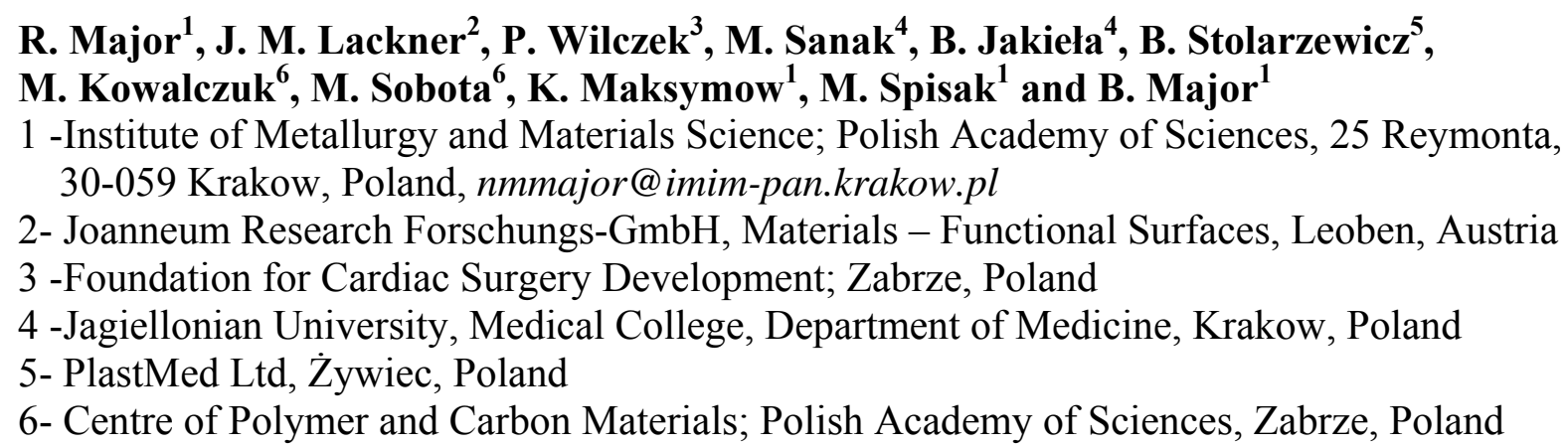

\title{
FUNCTIONAL CARDIO-BIOMATERIALS
}

\begin{abstract}
Titanium as well as carbon based biomaterials, seem to be good candidates for future blood-contact applications. Bio-materials such as: $\mathrm{Ti}, \mathrm{Ti}+\mathrm{DLC}, \mathrm{TiN} ; \mathrm{Ti}(\mathrm{C}, \mathrm{N})$ with higher carbon concentration and DLC (diamond like carbon) were under examinations. Trials on surface modification of PU (polyurethane) substrate using ion mill were performed. Materials were deposited as thin films by the hybrid pulsed laser deposition (PLD) technique to examine the influence of the fabricated surfaces on cell behavior. The metallic titanium as a target was used for titanium based-film and graphite one for DLC. Phase content of deposited films was controlled by the flowing gas mixture of $\mathrm{Ar}+\mathrm{N}_{2}$ and $\mathrm{Ar}+\mathrm{N}_{2}+\mathrm{C}_{2} \mathrm{H}_{2}$ type in the reactive chamber. Sputtering of graphite was carried out in Ar atmosphere. The kinetic energy of the evaporated particles was controlled by application of variation of different reactive and non reactive atmospheres during deposition. Transmission electron microscopy (TEM) was used to reveal structure dependence on specific atmosphere in the reactive chamber. The measurement of the strength of bonds between biomaterials and cells is a major challenge in cellular biology since it allows for the identification of different species in adhesion phenomena. The biomaterial examinations were performed in static conditions with Dictyostelium discoideum cells and then subjected to a dynamical test to observe the cell detachment kinetics. For a given cell, detachment occurs for critical stress values caused by the applied hydrodynamic pressure above a threshold which depends on cell size and physicochemical properties of the substrate, but it is not affected by depolymerization of the actin and tubulin cytoskeleton. Tests revealed differences in behavior in respect to the applied coating material. The strongest cell-biomaterial interaction was observed for the carbon-based materials compared to the titanium and titanium nitride.

A surface fuctionalization was realized by: (i) fabrication of migration channels by laser ablation, (ii) electrospinning and (iii) deposition of multilayer film from polyelectrolites. A goal of the performed research was formation of scaffolds for bio-mimetic coatings. Surface morphology examinations and biomedical studies on porous and semi-porous materials with application of human endothelial cells HUVEC line were performed by application of confocal laser scanning microscopy (CLSM).
\end{abstract}

Key words: pulsed laser deposition, microstructure, cell adhesion, surface functionalization

\section{INTRODUCTION}

Over the past 30 years, much effort in the research of biomaterials has been directed toward the development of materials that are inert to human blood and do not react with platelets or coagulation factors [1]. A number of often conflicting approaches have been developed. There is still no consensus about the advantageous hydrophilic or hydrophobic behaviour of a surface. This lack of agreement is largely due to both the incomplete understanding of the biological pathways leading to material failure and the designers' 
inability to fully evaluate the blood material failure and the blood material responses. In the development of the materials with a lower thrombogenicity, researchers have primarily focused their efforts on modifying the surfaces. This approach is reasonable, since it is only the surface chemistry of the material that should dictate its biological responses and the mechanical characteristics of the material are primarily dictated by the bulk chemistry. Despite the successes in reducing the protein and the cellular deposits on some materials, a truly nonthrombogenic surface does not exist.

Experimental and theoretical work has been dedicated to the cell adhesion from three very different perspectives [2-8]: (i) From the biological point of view, the understanding of the molecular mechanisms is of central importance, which means the understanding of when a cell adheres, rolls, or slides on passive or reactive substrates. Many functions performed by living cells depend on these properties. (ii) From the physical-chemical point of view, bioadhesion involves cells, a solid substrate, and a liquid medium. The relevant properties of a micro organism are the hydrophobic and the charge of the cell surface, cell size, and possession. A lot of experimental and theoretical work has been dedicated to cell adhesion [3]. (iii) From the physical point of view, even the passive (non-reactive) response of the cells to an external force offers new phenomena that find no equivalent in more traditional materials. This is the case in the bio-adhesion phenomena, where the discrete nature of the contact regions, the weak or non-covalent bonding of the cell to the substrate, and the multicomponent variety of the cytoplasm membrane or of its extra cellular matrix (ECM) are the origin of contact forces. Measuring the strength of these bonds is a major challenge in cellular biology, since it allows for the identification of the different factors at work in the adhesion phenomena.

The topography of surfaces is an important property for the contact of a component to its surrounding. The tribological behaviour, the decorative appearance and even the interaction to biological matter (cells, bacteria, and tissue) is strongly influenced by the texture in macro-, micro- and nano-scale. Shape deviations, waviness, grooves, etc. are some of the macro- and micro-scaled topography features, while nano-scale features include the roughness due to the microstructure and due to the atomic arrangement (lattice). It's quite clear, that mainly the nano-scale features gain more and more interest for functional surfaces in sensors, electronics, medical and implant technology and mechanical engineering.

Nanostructures between atomic and micrometer size (generally between 1-100 nm) can be achieved by a multitude of techniques, developed since the early 1990s. Different approaches are used both in laboratory and in industrial scale to generate nanostructured surfaces [9-14].

Surface treatments (physical and chemical surface modification) can be effectively done by plasma techniques to shape the surface for cell-specifically focal adhesion spots of several nanometers diameter. The formation of this biomimetic nano-topography and surface chemistry can be performed on the whole scaffold surface (including the open porosity), which is a novelty in plasma technology. Using the materials under examinations prepared by the authors [14], subsequent examinations on hemocompatibily were performed by Sanak et al. [16] by application of a simplified experimental setup, based on a commercial cone-andplate analyzer. Information are given which seems limiting usage of artificial materials in contact with blood.

The results presented in this work concern the problems of materials science at the intersection of engineering and medicine and precisely, the surface characterization and the cell-material interaction of thin films in the detachment assays. A surface fuctionalization was realized by: (i) fabrication of migration channels by laser ablation, (ii) electrospinning and (iii) deposition of multilayer film from polyelectrolites.

A goal of the performed research was formation of scaffolds for bio-mimetic coatings. 


\section{MATERIALS AND METHODS OF EXAMINATIONS}

\section{Preparation of the surfaces and diagnostics}

A development of the surface modification with the use of thin film materials was performed by hybrid PLD and mostly by magnetron sputtering in direct current (DC), unbalanced mode. Titanium (medical grade titanium for titanium and titanium nitride coatings) and carbon targets (for Ti(C,N) and DLC) were used to deposit about 10-20 nm thick films on polyurethane substrates at room temperature in argon atmosphere (Ti) or nitrogen-argon atmosphere (TiN). To ensure the homogenous film thickness over the entire coated surfaces, the substrates were rotated during the deposition at the speed of $5.4 \mathrm{~cm} \mathrm{~s}^{-1}$ through the plasma plumes. A detailed description of the deposition arrangement is given elsewhere [10-15].

\section{Thermal effect}

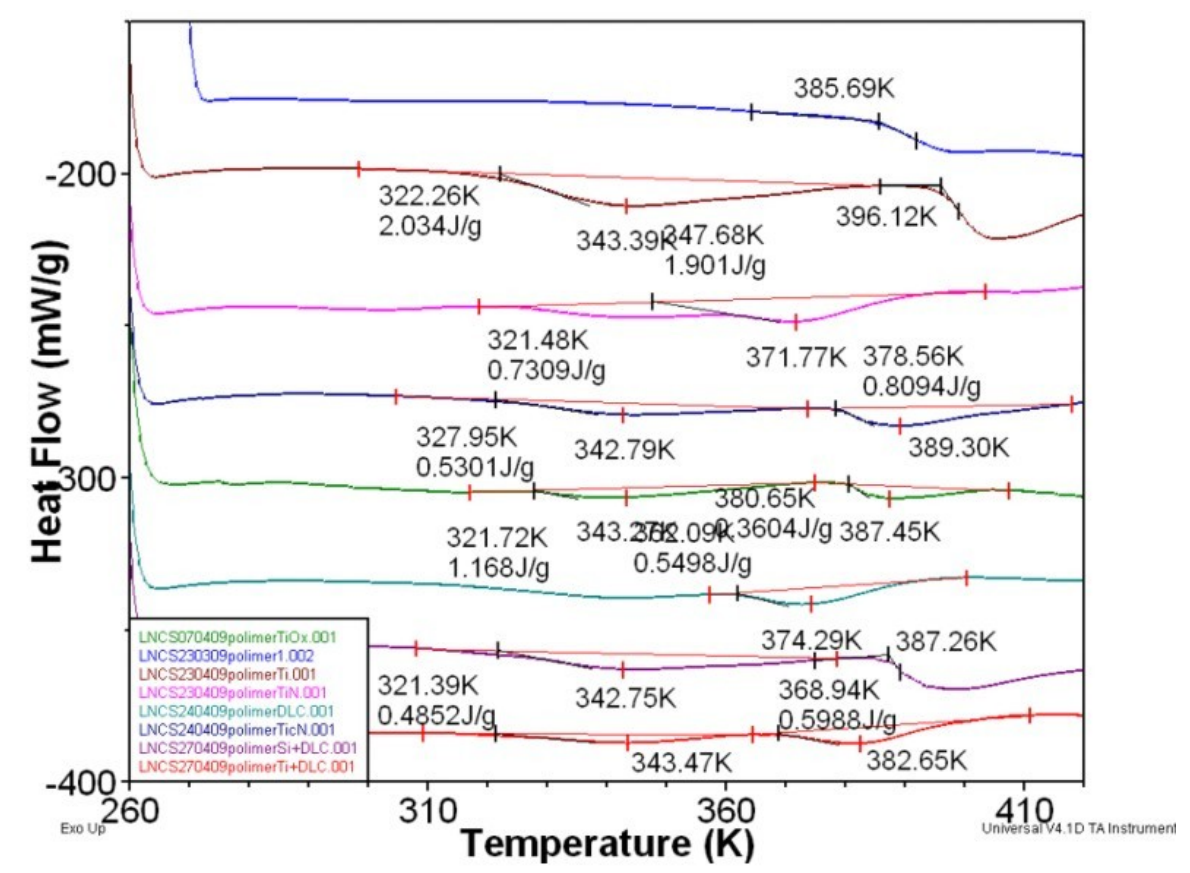

Fig. 1. Heat Flow in function of the applied temperature in calorimetric measurements for coatings deposited on PU: (from bottom) Ti+DLC, Si+DLC, Ti(C,N), DLC, TiN, Ti, polymerX, TiOx

Deposition process could have the significant influence on the material properties [17]. It is a crucial aspect in the case of biomaterials designing procedure. The temperature and the atmosphere seem to be the basic parameters which determine the proper structure. The temperature effect was analyzed as the Heat Flow in function of the applied temperature (Fig.1). The performed investigations involved a small glass transformation on the level of 110 deg C. All the visible effects probably come from the substrate. The following materials which were applied as the surface modification exhibited various reactions:

$-\mathrm{Ti}, \mathrm{Ti}(\mathrm{C}, \mathrm{N}), \mathrm{TiOx}, \mathrm{Si}+\mathrm{DLC}$ caused an additional endothermic effect in the lower temperature area,

-TiN, DLC, Ti+DLC influenced the additional endothermic effect but the change of the temperature of the glass transformation is visible as well. 


\section{Surface analysis}

The surface analysis was performed using a scanning electron microscopy (E-SEM) and a scanning acoustic microscopy (SAM) [14]. The proper surface structure and the lack of cracks is strongly correlated with the appropriate mechanism of the thin film growth [13]. The coating deposited under the late mechanism of the thin film growth is brittle and exhibits the cracks on its surface [11].

\section{Scanning electron microscopy E-SEM and Confocal laser scanning microscopy CLSM}

In the E-SEM and CLSM examinations of Ti, TiN, Ti(C,N) (Fig.2), it was stated that these films have the proper thickness. The visible scratches come from the substrate. The titanium coating was too thick. It caused numerous cracks, which could influence the platelet activation process. Thus, the thrombus formation could be blurred by the effect coming from the scratches. An optimization of the deposition parameters leads to the limiting of the thickness of the film to the primary mechanism of the deposition [13].

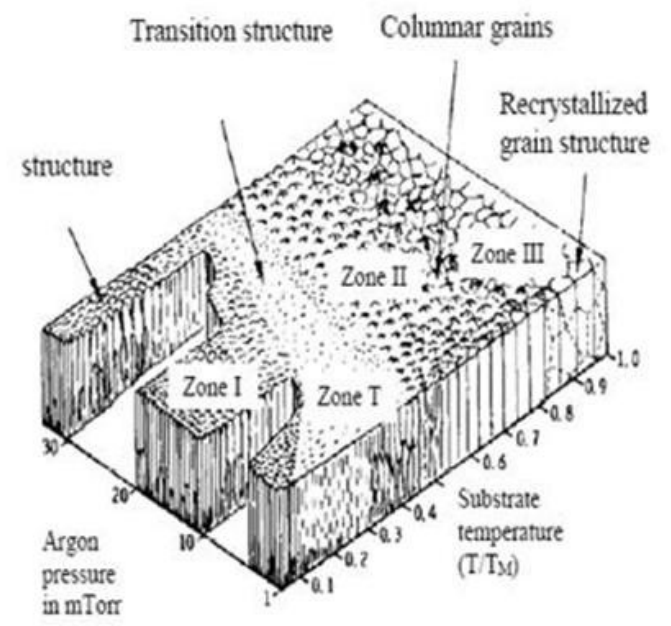

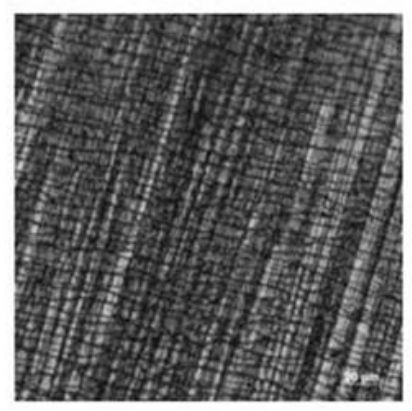

$\mathrm{Ti}$

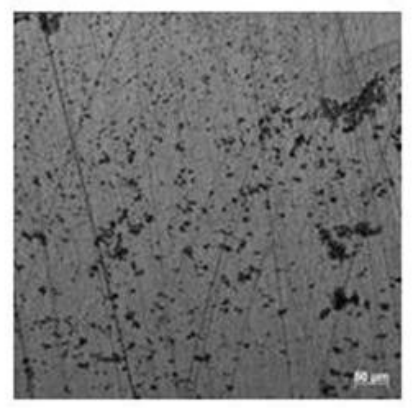

TiN

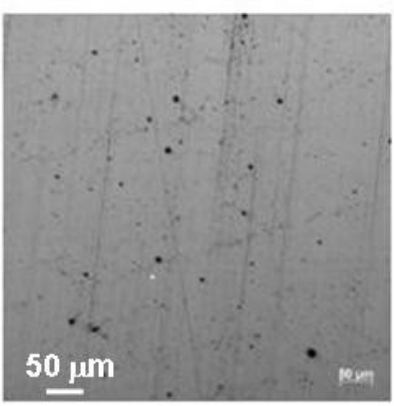

$\mathrm{Ti}(\mathrm{C}, \mathrm{N})$

Fig. 2. CLSM (confocal laser scanning microscopy) examinations of the deposited coatings with the Thornton diagram [17]; defects in cracking forms can be seen; more cracks in Ti coating; least defects in Ti(C,N), TiN

\section{Scanning Acoustic Microscopy SAM}

The Scanning Acoustic Microscopy (SAM) is a technique which enables to investigate whether the material has been uniformly or non-uniformly deposited [14,18-21]. The only problem which was observed was associated with Ti. The other coating materials were 
deposited properly. In the case of $\operatorname{Ti}(\mathrm{C}, \mathrm{N})$, a visible effect of the residual stress concentration was seen by the isolines appearance (Fig.3).

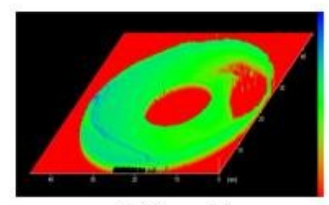

Ti_level 0

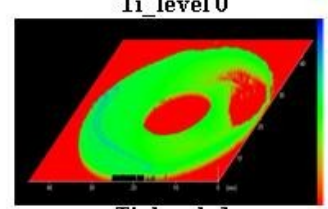

Ti_level-1

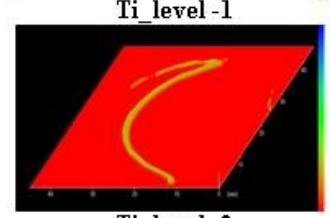

Ti_level-2

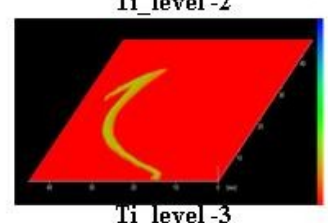

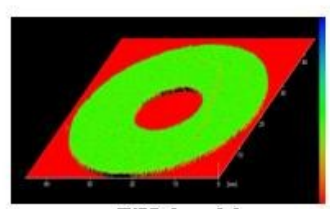

TiN_level 0

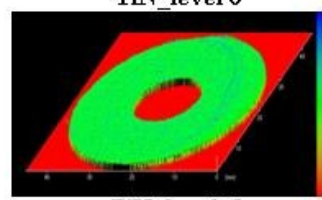

TiN_level-1

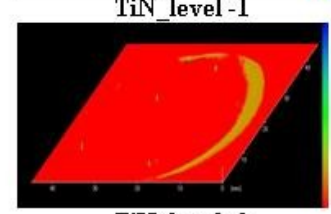

TiN_level -2

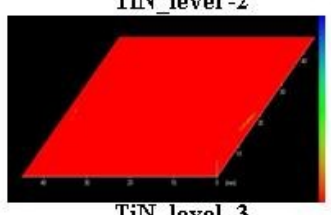

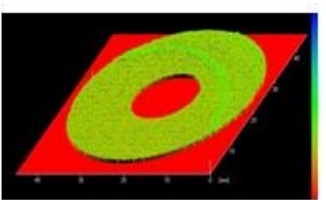

Ti(C,N)_level 0

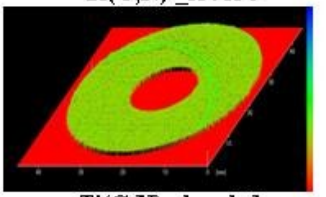

Ti(C,N) _level-1

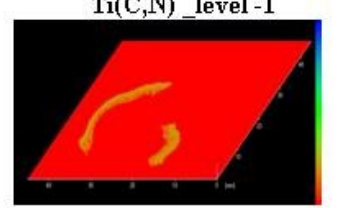

Ti(C,N)_level -2

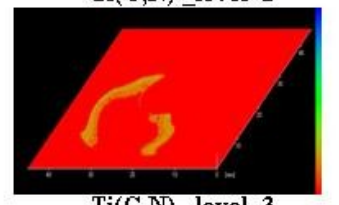

Ti(C,N)_level -3

Fig. 3. Tomography analysis of Ti, TiN and Ti(C,N) coatings using SAM (scanning acoustic microscopy) on different thickness levels, starting from the surface to bottom of coating at thickness step of about $7 \mathrm{~nm}$; thickness of coating about $20 \mathrm{~nm}$; area of adhesion (light contrast); pictures of $0,1 / 3,2 / 3$ and 1 thickness of coating

\section{Microstructure analysis}

The intended target of the work was to elaborate materials which would not change the properties of the PU substrate but also effectively separate it from the tissue.

The transmission electron microscopy TEM and the high resolution electron microscopy HREM was used to study the microstructure of the thin foils prepared from the film crosssection, using the focused ion beam (FIB) technique $[12,14]$. The examinations focused on the study of the adhesion of the coating to the substrate and thus, the quality of the bio-material (Fig.4). In detailed TEM examinations. the microstructure of Ti presents columnar character which proves the late mechanism of the thin film growth It could result in the stiffness increscent of the PU-coating system which automatically would reject such material from the clinical use. Cross-section in the bright field mode of the $\mathrm{TiN}$ and $\mathrm{Ti}(\mathrm{C}, \mathrm{N})$ microstructure, deposited on PU, were similar and exhibit elastic properties of the coating. This was possible due to the proper mechanism of the film growth leading to close an amorphous structure. 


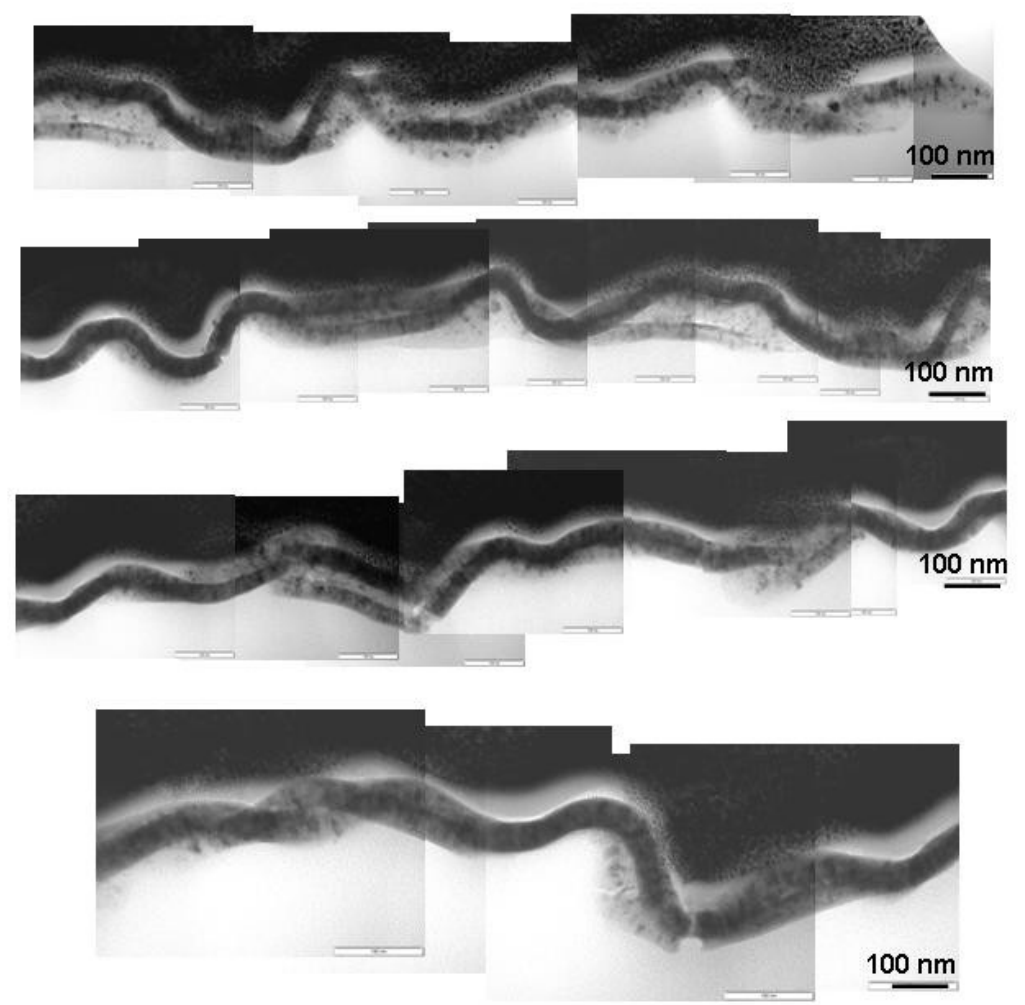

Fig. 4. TEM (transmission electron microscopy) bright field of TiN/PU coating, cross-section, sets of pictures along the coating

\section{PREPARATION OF THE CELL CULTURE}

\section{Cell culture}

For the study of the cell-material interactions, the mouse fibroblast cells line L929 from the ATCC collections were used. The cells were cultured in "Medium 199" supplemented with 10\% FCS (Fetal Calf Serum) and with antiobiotics streptomycine and penicyline (Sigma) at $37{ }^{\circ} \mathrm{C}$ with $5 \% \mathrm{CO}_{2}$ in a humidified incubator (Haereus). The media were changed every 2 days. The cells were cultured in $25 \mathrm{~cm}^{2}$ culture flasks. The media were changed every 2 days. When the L929 cells in the culture had grown to $75 \%$ confluency, the cells were ready to use for the cytotoxicity tests [22].

\section{Seeding procedure}

Before the tests, the samples were subjected to the sterilization process using ethylene oxide. Afterwards, they were ventilated for 2 weeks to remove the rest of ethylene oxide. Before seeding, the cells were harvested from the culture dishes using 0,05\% Trypsin/EDTA solutions and resuspended in the culture medium (Medium 199 supplemented with 10\% FCS, $1 \% \mathrm{~L}$-glutamine) to the concentraction of $5 \times 10^{6}$ cells $/ \mathrm{ml}$. 
The tested materials were cut into squares and placed in 24 wall culture dishes. To ensure a complete contact between the samples and the wall, the samples were pressed with plastic rings. Then the pellet of the resuspended cells were plated on the material surface with the density of the cells of around 10000 cells/well. After $30 \mathrm{~min}$. of incubations, the culture media were completed to a total volume of about $3 \mathrm{ml} /$ well. An empty well with fibroblast cells was used for control purposes. The materials with the cells and the control were cultured at $37^{\circ} \mathrm{C}$ with $5 \% \mathrm{CO}_{2}$ in a complete medium (Medium 199 supplemented with $10 \% \mathrm{FCS}, 1 \%$ L- glutamine) for a total of $24 \mathrm{~h}$.

\section{Fluorescent microscopy}

In order to observe the changes in the cell viability and morphology after the cell material interactions (attachment), we applied a fluorescent microscopy. The viability of the cells was analysed with the use of the Live/Dead dye Fluoresceine Diacetate (FDA) and the Propidium Iodide (PI). The viable cells stained green with FDA and the non-viable cells stained red with PI.

\section{CELL-MATERIAL INTERACTION}

\section{Cell-material interaction in hydrodynamic conditions (Radial detachment test) BIO kinetics - Cell detachment}

Dictyostelium discoideum was used as a model organism to study cell detachment kinetics [2-8]. In the case of $D$. discoideum a unicellular eukaryote also able to complete a pluricellular development cycle, membrane adhesiveness is tightly related to lifestyle. In the vegetative phase, the small $(8-\mu \mathrm{m}$ diameter) ameba feeds upon bacteria and yeast by phagocytosis. Plasma membrane adhesion is therefore directly related to the phagocytic properties of the cell. In the development phase, cell-cell adhesion is more important, and specific contact proteins are expressed [2]. Axenic strains showing an enhanced fluid-phase endocytosis have been obtained and are able to grow in suspension in nutritive medium [3]. The sequencing of $D$. discoideum 34-Mbp genome and Expressed Sequenced Tags from various developmental stages are currently in a completion phase [4]. The available molecular tools make $D$. discoideum a good model organism to study phagocytosis and cellular motility using null or overexpression mutants [3]. Several mutant studies suggested the existence of three kinds of adhesion protein. A glucose-selective lectin, mediating attachment of bacteria to the cell, was revealed by the sensitivity of mutants to $50 \mathrm{mM}$ glucose. A hydrophilic receptor called Phg1, consisting of a 9-helix transmembrane protein was recently identified. As for now, the molecular nature of its ligand is unknown, but it is present in nutritive media, because Phg1 null mutants are adhesion defective in this medium. The third class of the adhesion molecule is sensitive to surface hydrophobicity, as exemplified by the HV32 mutant, which does not bind to polystyrene in the presence of glucose. 


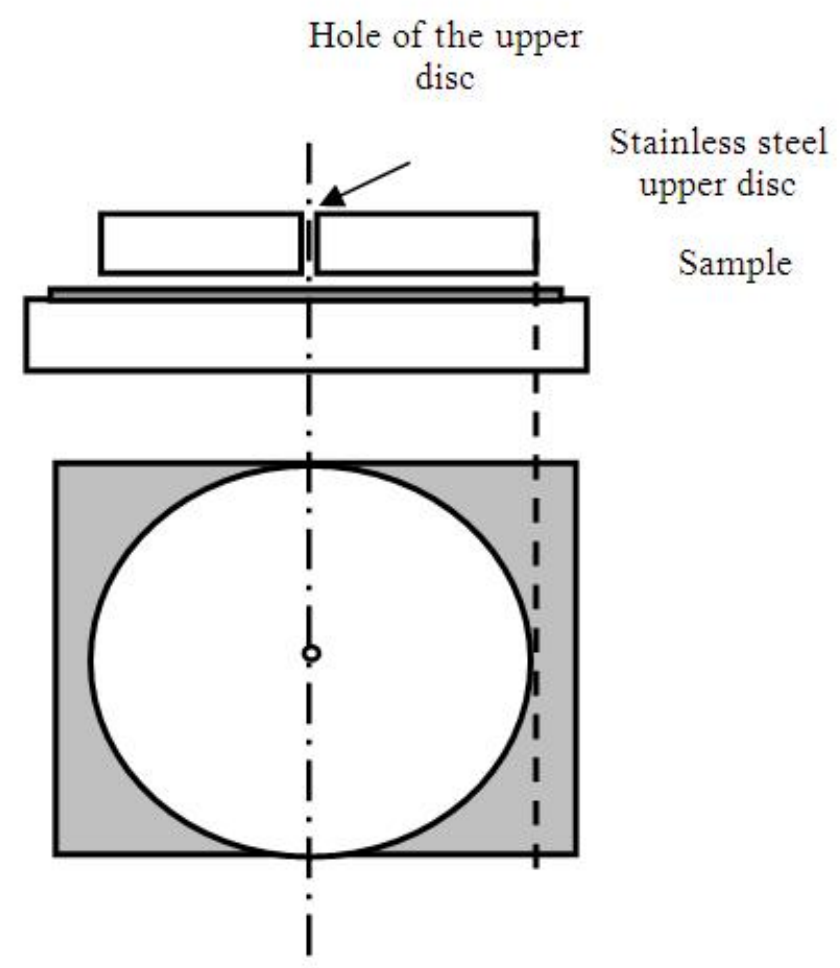

Fig. 5. Cell detachment assay equipment layout for radial shear flow test

For a given cell, detachment occurs for values of the applied hydrodynamic pressure above the threshold. In this purpose, a radial flow detachment essay was used. Cells were resuspended in the Sörensen buffer and spread at a density of 300 cells $/ \mathrm{mm}^{2}$ on the substrate surface and subsequently submitted to the shear flow under the radial geometry. The temperature of the liquid was kept constant at $21^{\circ} \mathrm{C}$. A radial hydrodynamic flow was generated between the stainless disc and sample on which cells adhered (Fig.5) [5-8]. The stainless steel disk with a hole in the center was placed above the sample with the $100 \mu \mathrm{m}$ distance. The action of the shear flow depends on the distance between the disc and the sample and the level of the upper tank influence on the shear stress between the cells and the material surface. The layout of the investigation is presented in Fig. 5. After the test, samples were given under the fluorescence microscopy observation. In use of Image-ProPlus software, the cells were counted and on basis of the received values critical shear stress was calculated in use of the following formula (1):

$$
\sigma_{50 \%}=\frac{3 D \eta}{\pi r_{50 \%} e^{2}}
$$

$r_{50 \%}$ is the distance to the center where $50 \%$ of cells are detached

$D$ - rate during the test, $\eta$ - buffer viscosity, e - space between disk and plate

In order to receive stagnation point, the considered time of the test changed from 5 to 10 minutes. It was observed that shear stress should be applied for longer times to determine detachment kinetics. Titanium nitride coating was exposing for the three deferent test durable, 
5, 7.5, 10 minutes. The longer the time is the more cells are detached. Comparing the results between the substrate and the coating, $20 \%$ more cells remained on the uncoated substrate.

In conclusion to this result shear stress should be applied for longer times to determine detachment kinetics. It was observed no clear difference between 5 and 10 min, thus steady state is almost reached.

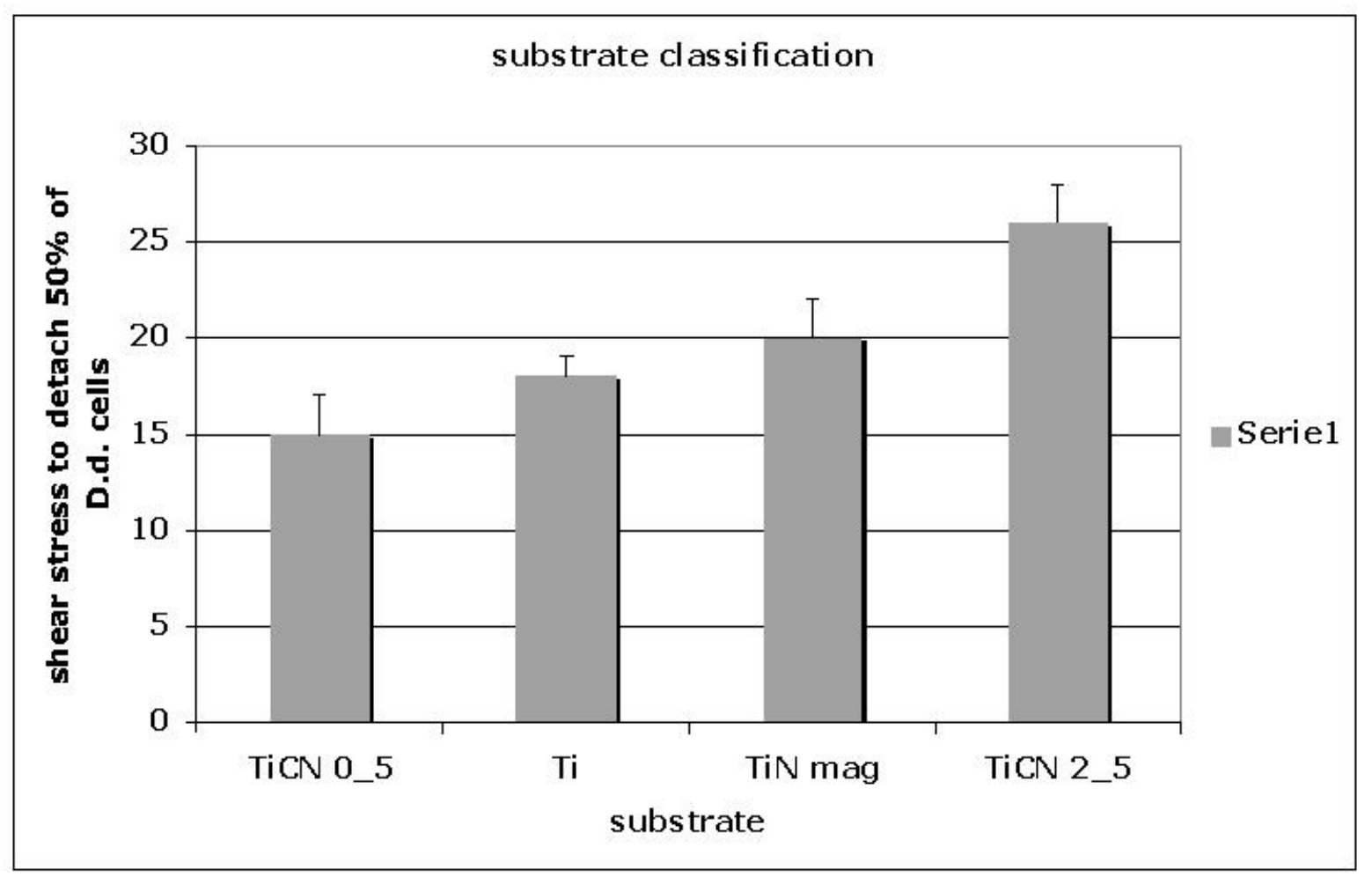

Fig. 6. Shear stress classification for: Ti, TiN, Ti(C,N) low C / indication on figure TiCN 0_5/ , Ti(C,N) high C / indication on figure TiCN 2_5/ (serie 1)

In summary, the total values obtained in these series of experiments of the shear stress were collected and compared. Fig.6,7 present a total classification of the shear stress which was detected between the coating and the biological cell.

The percentage of detached cells was recalculated as a function of shear stress, since not so many cells were detached, the initial density from the distribution of remaining cells was taken. The center of the distribution was always between frames 190 and 205. The value that makes the distribution symmetrical was put. Sometimes, the distribution was not symmetrical. The center and the percentage of detached cells was shown as a function of shear stress. Data obtained for 10 min flow to data obtained after shorter flow duration were compared. In Fig.6 there are shear stress ordered as a function of material: The less adhesive one is TiCN 0_5; the order is as follow: TiCN 0_5 $<\mathrm{Ti}=\mathrm{TiN}$ magnetron $<\mathrm{TiCN} 2 \_5$.

The radial shear flow test was used to determine the efficiency and the kinetics of the cell detachment from the plain, as well as the modified surfaces as a function of the applied shear stress $[2,3,15]$. The investigated materials were considered in the particular conditions described above. Depending on the material, a different exposition time was necessary. The results of the cell efficiency and cell rate detachment under the applied shear stress were also studied. TiN exhibits a low cell material interaction. The detachment rate for titanium carbonitride with a low carbon content was over estimation. The values of the threshold stress 
confirm the observations of the cell distribution. The lowest value 3Pa typical for TiN, the highest for TiCxNy(highC) with a high carbon content $7 \mathrm{~Pa}$. The function extrapolation for the $0 \mathrm{~Pa}$ hydrodynamic stress revealed a spontaneous cell detachment rate; $0.02\left[1 / \mathrm{min}^{-1}\right]$ for $\mathrm{Ti}(\mathrm{C}, \mathrm{N})$ and $0.008\left[1 / \mathrm{min}^{-1}\right]$ for TiN [15]. The cell detachment test gives an overview of the cell behaviour and the shear stress which could appear between the cell and the biomaterial. The biomaterial design requires biophysical tests, as well as biocompatible tests which would illustrate the biological response of the biomaterial to the biological cell. In this case, the tests are performed in static conditions on fibroblas.

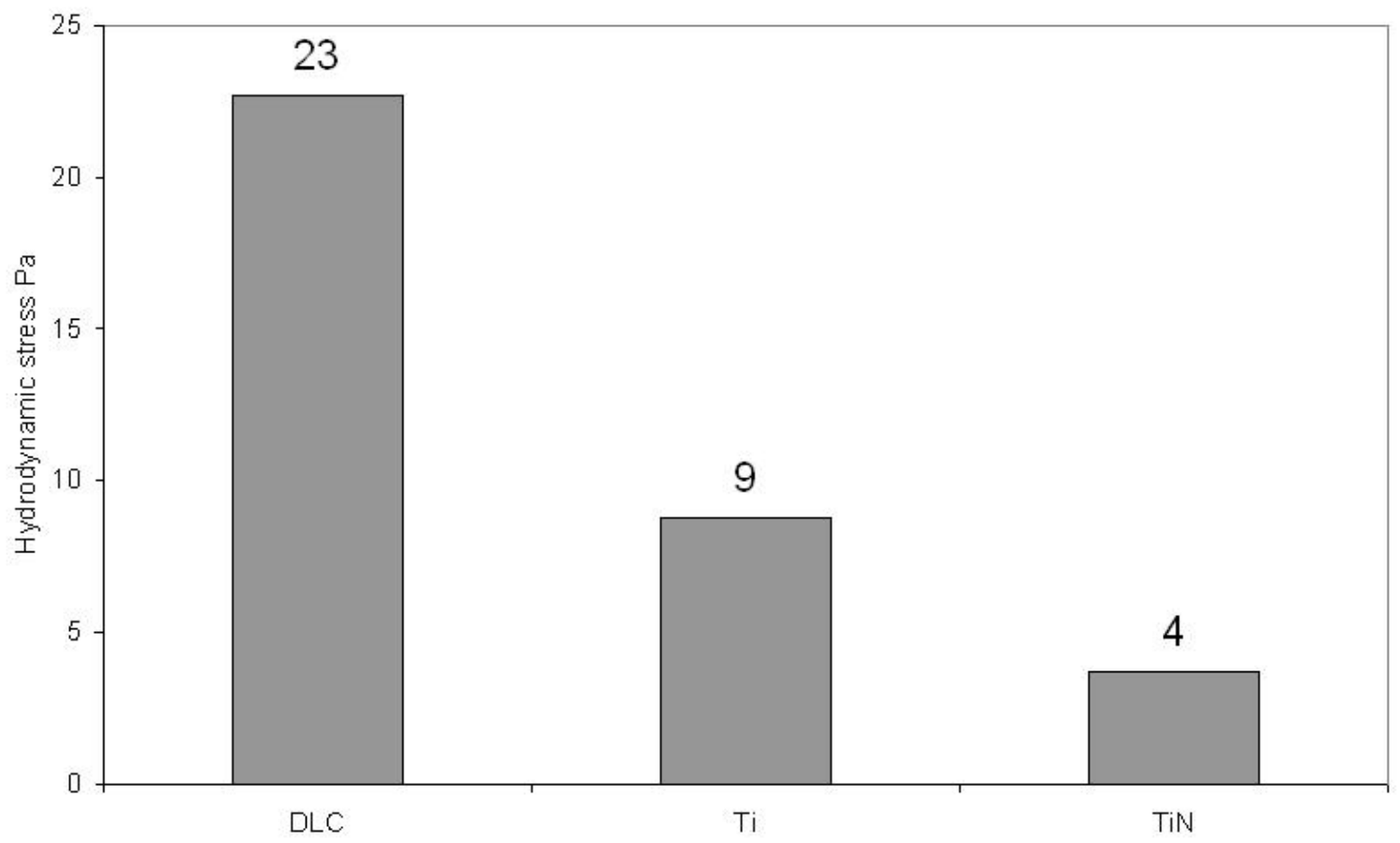

Fig. 7. Shear stress classification for: DLC, Ti, TiN (mean values of a few series)

\section{Cell-material interaction in static conditions (biocompatibility testing)}

The presented sub-chapter describes the biocompatible tests performed on the investigated materials in static conditions. Two important aspects of the biomaterial design were considered - the stoichiometry and the phase composition modification with an introduction of carbon. To measure the biocompatibility, fibroblasts were applied.

The stoichiometric titanium nitride is known as a biocompatible material. The lack of stoichiometry could result in a negative influence on the surrounding tissue. In order to examine this problem, both groups, the biocompatible and the non-biocompatible titanium nitride, were studied. Fig. 8 presents the result of the biocompatibility tests of the example material. For this purpose titanium nitride (TiN) was considered.

The experiments revealed that the cells exhibit a high biocompatibility on the surface of the fully stoichiometric TiN (Fig.8a). There was a visible growth in the cells and a spread across the surface and no influence on the cell death was detected. The cells revealed individual focal adhesions.

The cell function is also related to the cell shape [1]. The cells grow when spread, die when fully retracted, and differentiate into capillary tubes if maintained at a moderate degree of extension, while also forming cell-cell contacts (e.g., on thin micro patterned lines). 
On the non-stoichiometric titanium nitride, the cells demonstrated round shapes, typical for the cell death (Fig.8b). Over $50 \%$ of the deposited cells were directed to the death path. The remaining live cells did not spread on the surface.

As presented in Fig.8, the stoichiometric titanium nitride exhibits good biocompatible properties. When designing a new biomaterial, a phase composition modification by means of carbon was considered. A comparison of the biocompatible results for the stoichiometric titanium nitride, as well as for the titanium carbonitride with a low and high carbon content has also been studied. .

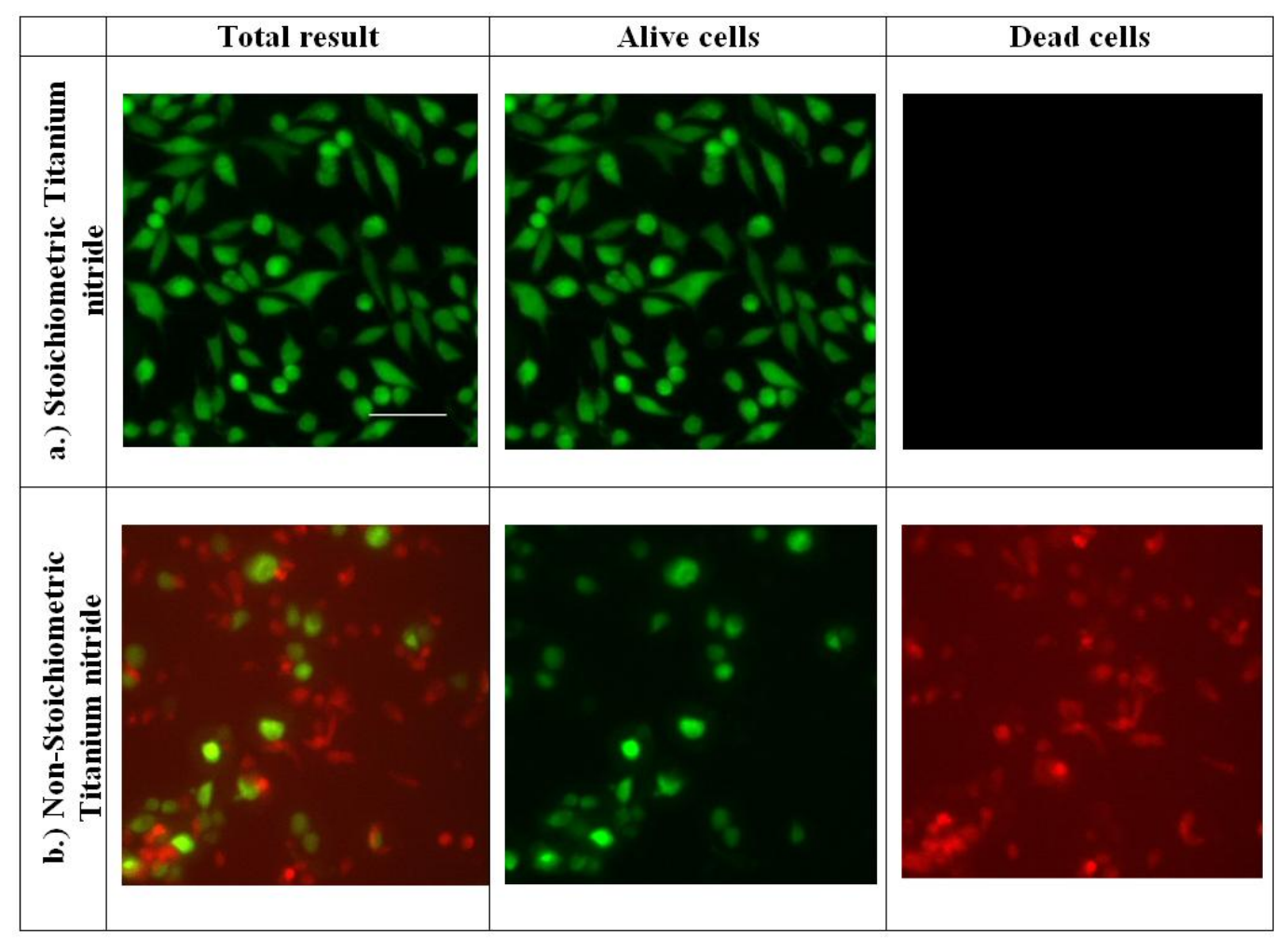

Fig. 8. Material biocompatibility (fibroblasts), a) Cells reaction on stoichiometric titanium nitride;

b) Cells reaction on non-stoichiometric titanium nitride

\section{PRINCIPLES OF TESTS FOR HEMOCOMPATIBILITY OF MEDICAL MATERIALS}

International Organization for Standardization (ISO) developed a guidance on testing medical materials that have contact with circulating blood (ISO 10933-4). In brief, a hemocompatible material must not adversely interact with any blood components [22]. A measure of this adverse interaction can be inappropriate activation or destruction of blood components. The guidelines provide a structured selection of methods useful in testing for interactions with blood. It is based on the intent to use of the material, however, it does not provide the exact test methods or evaluation criteria. Instead, a list of various applicable references is suggested.

Blood is a complex tissue, comprising plasma and cells. Blood plasma is the isotonic electrolyte solution corresponding to $0.9 \%$ sodium chloride, with relatively high protein content (40-60 g/L). Albumin makes more than half of total plasma proteins, but many low 
level constituents are critical for the function of whole organism, e.g. clotting factors, antiproteases, transporter proteins, or immunoglobulins. The most abundant blood cells are erythrocytes, which extremely high count of 4 million per one $\mu \mathrm{L}$ causes that $40 \%$ total blood volume is occupied by erythrocytes. These cells are also the most rigid ones and prone to rupture and subsequent hemolysis, which can also occur upon the change in osmotic pressure, or interaction with mechanical devices. Blood platelets are roughly twenty times less abundant and platelet diameter is only the one fifth of erythrocyte. Blood platelets are critical for vascular homeostasis, as they easily activate in contact with the exposed components of the vessel wall, granting protection for vascular bed integrity and sealing of any blood leaks. This primarily hemostatic function of platelets could lead, in certain conditions, to the thrombosis. It is also one of the major obstacles in the use of biomaterials that contact with blood. Hemocompatibility categories pointed out by ISO guidelines refer to several aspects of blood physiology, as: coagulation, platelet status, thrombosis, hemolysis and activation of innate immunity complement proteins. Thrombosis occurs normally only at site of vascular injury and involves platelet adhesion followed by the activation of blood clotting cascade and formation of fibrin clot. Though blood platelets are a major morphotic component of thrombus, other cells, like erythrocytes and leukocytes can also accumulate at the site of fibrin deposition. Activated leukocytes easily adhere to the surface of endothelial cells, and migrate out of bloodstream into surrounding tissues. This property is also important for testing of hemocompatibility, as these cells can activate alongside the platelets and adhere to the vascular wall promoting thrombosis.

Commonly used test conditions mimicking vascular flow aim to simulate dynamic interaction between whole blood and biomaterial [22-24]. Dynamic test differs from a static interaction by introduction of shear forces activating blood cells. Shear stress, which is measured in units of pressure, is expressed more conveniently as the shear rate, which changes linearly with the stress, providing constant viscosity of blood. From technical point of view, materials are tested using flow chambers of a different design. Blood used in these tests is anti-coagulated with sodium citrate or heparin to prevent clotting. To avoid artifacts, dynamic tests for hemocompatibility are preferably performed using one-pass of blood and their duration ranges usually from 2 to 5 minutes but can be extended up to 1 hour.

\section{Method to assess hemocompatibility in high shear stress conditions}

The study was designed to evaluate performance of an alternative in vitro dynamic test for hemocompatibility. Instead of a linear flow chamber, the test is based on the cone and plate analyzer, an instrument validated for clinical use in evaluation of thrombotic diseases and the effectiveness of anti-platelet drugs. The rationale for this trial was: 1) lower requirements for donor's blood to perform the test, 2) availability of a commercial testing instrument, 3) accepted principle of a rotating cone and plate as a source of shear stress, as it is used in rheometers or viscometers. The study design allowed also to test inter-day variability of a single donor blood, to ascertain its impact on the reproducibility of results. The following research was performed:

- Flow cytometry analysis of the blood

Two sets of tubes were prepared for each tested plate and for the static controls. Expression of platelet activation markers was ascertained using whole blood staining.

- Measurements of thrombogenic microparticles activity

Thrombogenic potential of blood plasma was measured using Zymuphen MP-activity ELISA kit (Hyphen Biomed, Eragny, France), according to the manufacturer instruction. This 
assay is based on trapping of phospholipid-rich microparticles derived from cell membranes, using immobilized annexin $\mathrm{V}$, followed by reconstitution of thrombin activity with calibrated clotting factors solution. Proteolytic activity of generated thrombin against the chromogenic substrate strictly correlates with concentration of microparticles present in blood plasma [27].

- Statistical analysis

Statistical analysis was performed using SPSS version 17.0 statistical package (SPSS Inc., Chicago, IL, USA) for personal computers. Data were studied as means \pm SD (standard deviation) and ranges. Statistical significance was assumed for type-I error $p<0.05$.

- Platelet aggregates formation during the shear stress

- Platelets activation following the shear stress

- Platelet - granulocyte aggregates

- Plasma thrombotic potential resulting from the formation of platelet microparticles

- Ranking hemocompatibility of tested materials

A relative rank for hemocompatibility of tested materials was constructed for a study using 2-dimensional plots, where percentage of platelets remaining after the shear stress was plotted against: platelets aggregates, platelet-monocyte aggregates, platelet percentage positive for activation marker P-selectin and concentration of microparticles in blood plasma. The lower right corner of these scatterplots theoretically represent the most favorable properties of the test material, i.e. no consumption of platelets and the lowest value of the parameter for adverse platelet function. It is noteworthy, that some materials, e.g. TiO performed the worst using either of the parameters, while some other gave results which differed diametrically, for example TiN and TiCN. These two materials substantially activated platelet markers but had the lowest generation of thrombotic activity related to microparticles.

\section{Discussion of tests for hemocompatibility of medical materials}

In vitro testing for hemobiocompatibility remains a challenge because the background of interactions between blood and foreign material is not fully explained [28]. It is generally acknowledged that initial and rapid adsorption of plasma proteins can establish further biocompatible properties of the material [29,30]. The process can be quite selective, as evidenced for PS surface, which covers with large proteins, including von Willebrand factor, a major docking molecule for platelet. From the technical point, quality of the material surface is also very important. During blood flow, any rough surface, its protuberances or cavities, can result in non-laminar flow, and precipitate cells activation and adhesion. Physiological endothelium has very smooth surface, because the cells are flattened to much less than $1 \mu \mathrm{m}$ thickness. The major limitation of in vitro tests for hemocompatibility is due to a short time of the experiment. Blood is a live tissue and viability of platelets deteriorate with storage time longer than 60 minutes along with their spontaneous activation. Moreover, conventional test chambers with laminar flow require volumes of blood which frequently exceed $100 \mathrm{ml}$ during 5 minutes experiment. Inter-individual and inter-day variation of peripheral blood morphology parameters is high and in our observation done on carefully selected donor exceeded 24\%. Drugs affecting platelet functions, like non-steroidal antiinflammatory drugs (e.g. aspirin) readily available over the counter, also have to be taken into consideration. With all these factors contributing to the high variation in the experiments for testing hemocompatibility, we decided to simplify in vitro setup of the test by using a well established rotating cone and plate viscometer. ISO gudlines for testing hemocompatibility insist on the usage of standard methods [22]. In line with this, we adopted a commercial instrument, marketed for clinical diagnostics of platelet dysfunction, cone-and-plate(let) 
analyzer [31]. We also selected conventional biomarkers used in hemocompatibility tests, i.e. platelet destruction, platelet aggregates, granulocyte-platelet aggregates and platelet activation markers. With all of these we demonstrated feasibility to perform the tests. The major advantage of the setup was a low blood volume required for the test and the possibility to study several materials in parallel. A major limitation of this study was (i) a low statistical power due to the insufficient number of replicates of the materials studied, except PS and PU, (ii) the fact that the study was not controlled for the mechanical quality of the surface (except PS), as was demonstrated later during a microscopic analysis of the plates. Some of tested discs had surface defects in the form of corrugations, or cracks in the layered materials, (iii) microscopic analysis of adherent cells or protein deposits was not performed during the study. Nevertheless, some valid conclusions can be drawn from the current study. Among the studied parameters for hemocompatibility, total number of platelet aggregates should be split into small and bigger aggregates. The smaller aggregates comprising only few platelets adhering together probably mark for the mechanical quality of the surface and result from a moderate activation of platelets. Small aggregates always increase following the shear stress and the increase can be higher using rough surfaces. Bigger aggregates were observed only with some plates tested and can indicate two opposite processes. Increased number of bigger aggregates is observed with higher activation of platelets, but adherence of these aggregates to the material surface makes this marker incredulous. Similar comments apply to the markers of platelet activation. Lack of adherence of activated platelet to the test surface can explain contradictory results of TiN and TiCN tests. These materials showed a quite high expression of P-selectin and PAC-1 activation markers, but microparticles activity in blood plasma was among the lowest. Microparticles activity of blood plasma, which in our in vitro tests has reflected fragmentation of platelets and release of cell membrane vesicles during exocytosis seems to be a novel finding of this study. Microparticles impose thrombotic burden to circulating blood [6] and were recently found to correlate with atherosclerosis and risk for major adverse cardiovascular events, like heart infarct or stroke [32,33]. Though generation of platelet microparticles was noted using flow cytometry almost two decades ago [34], using in vitro models of whole blood activation, only recently thrombotic function has become measurable with modern ELISA assays [27]. This parameter, correlating with a clinical prognosis for the patient, seems of the highest importance in testing hemobiocompatibility of biomaterials. It is also one of the markers, which can be used in monitoring performance of cardiovascular protheses, since it can be measured in vivo.

\section{FUNCTIONALIZATION OF BLOOD CONTACTING SURFACES}

Even with continued advances in endovascular technology, surgical replacement of the disfunctional vessel by autologous grafts or synthetic prostheses remains the key therapy. Novel vascular grafts which provide higher patience rates, are therefore urgently required.

In recent years, new techniques have been developed to apply micro- and nano-architecture designs mimicking the three-dimensional morphology of the vessel wall, including bioactive surface structuring and in-vitro-manufacturing of cell-formations and tissue-engineered grafts. Using these new available technologies, the research activity is performed in two main directions by the authors of this contribution:

- Nanoscaled functionalization of porous scaffold biomaterials for cardiovascular prostheses (Fig.9)

- Nanostructural materials for implants and cardiovascular biomedical devices 


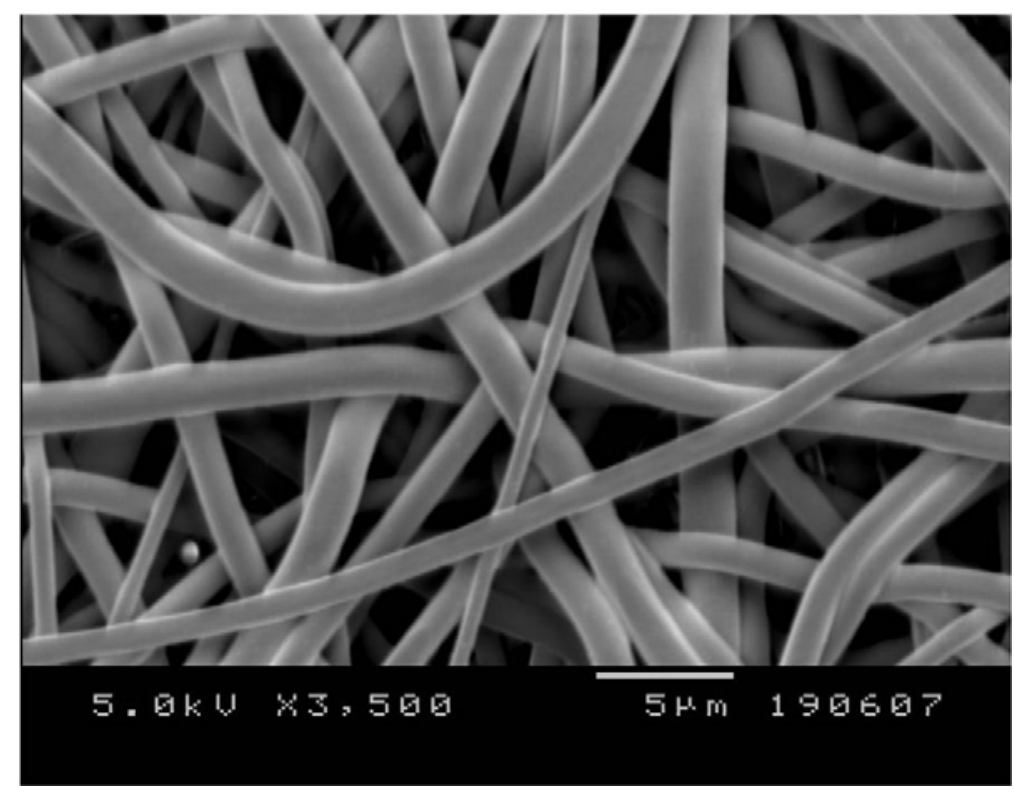

Fig. 9. Porous polymer scaffold for precursors of tissue analogs fabricated using electrospinning

The main objective of the research activity is the modification of polymer implants and tubes (cannula, conduits) on the external and internal surface for improving biocompatibility of tissue contact surfaces, especially in contact with blood. New, tissue contacting materials with main focus on blood contact aspects, for external and internal blood circulation support devices are planned to be elaborated. The surfaces of the implant devices will be covered with non-cytotoxic and anti-thrombogenic coatings biomimeticly behaved to its surrounding (e.g. vessel surface), which means to influence the endothelial cells to cover. Depending on the topography of the vessel inner-simulating surface and the chemical nature of the cells nonthrombogenic bio-surface, basing on cells covered polymer would be formed. Research is in progress to apply carbon, titanium and biopolymer based materials. Therefore, it will be used plasma pre-treatment by vacuum coating techniques. State-of-the-art devices are polymer tubes for forced external and internal blood circulation internally modified with heparin bond, preserving against the clot formation in blood contact. By lowering of the thrombogenicity, the increasing risk of bleeding is the main disadvantage of these systems. Thus, one concept is to improve the surface-blood cell interaction by using biomimetic (bionic) designs. In the case of implants in contact to muscle tissue the prevention of fibrosis is main topic.

Problems with current prosthesis systems (e.g. bypasses) are connected to intimal hyperplasia (occlusion of vessels by thick fibroblast deposits) after surgical implantation. Generally, the biomaterial acceptance and integration is dependent and controllable by the chemical composition and topography of the surface as well as by the mechanical properties of the bulk. Although cells are on the micrometre scale, surface modifications on the nanometre scale are of high interest, because all cell functions (protein adsorption, cell adhesion /orientation / migration) are based on interactions on the nanometer range (e.g. focal adhesions). For artificial small vascular prostheses, the most advanced and versatile technique for fabrication of the scaffold (support for tissue) is the electrospinning process (Fig.9), while large diameter prostheses used in clinics are knitted, woven or expanded today. Electrospinning allows an optimized control of microstructure (flat scaffolds with inherent 3D structures and macroscopic 3D shapes (tubes)) and fiber diameters in the micron range and below, all mimicking the structural properties of the biological extracellular matrix (Fig.9). The excellent mechanical properties of electrospun fibres impose micro-stresses necessary for 
maintaining the right cell phenotype. Control of pore size and distribution in the micron range allocate for space for extracellular matrix and cell metabolite production. Technological problems in electrospinning are connected to production reliability and performance, which must be optimized by jet control for future large scale industrial application. However, main task will be the reduction of thrombosis and intimal hyperplasia during clinical use of vascular grafts (which is currently the predominant restriction in clinical application of all vascular scaffolds). Attempts are both manufacturing improvements in materials (nondegradable, bioresorbable), and fiber alignment of scaffold fabrics (mechanical properties /stress) on the micro- and surface treatment on the nano-scale.

Surface treatments (physical and chemical surface modification) are effectively done by plasma techniques to shape the surface for cell-specifically focal adhesion spots of several nanometers diameter. The formation of this biomimetic nano-topography and surface chemistry is planned to be performed on the whole scaffold surface (including the open porosity), which is a novelty in plasma technology. We plan plasma etching, plasma deposition of organic and inorganic films (e.g. Si- / Ti doped diamond like carbon) and plasma grafting with functional molecules (e.g. amine, carboxyl groups), respectively. Both, full chemical / topographical homogeneity and gradients are expected for targeted protein as well as endothelial and smooth muscle cell adhesion (colonization) in- and outside the tube.

One of the important examination directions within precursors of tissue analogs is cell migration. A goal of such examination is targeted on contribution of an artificial surface to adhesion and direction of cell migration. A possible solution of this problem could be found in preparation of migration channels (Fig.10). They have been produced using the laser ablation technology. For an initial model analysis of cell migration, Dictyostelium discoideum cells were applied and subsequently studies on HUVEC line human cells (Fig.9-12) have been performed. Experiments were performed in static conditions in a vacuum flow chamber.

Both strategies of material development require advanced material and biomedical invitro and in-vivo testing. Materials testing will include both the development of quick, reliable, simple test methods for future manufacturing control and the scientific description of topographical and chemical changes on the surfaces. Biomedical in-vitro testing methods will focus both on short- to long-term protein adsorption and cell adhesion (single cell phenotypes and whole blood). In-vivo testing is planned to be performed in animal studies in the final stage.

Concluding, the research work with the migration channel could have the only basic science character. The process is complicated and expensive. As an advantage one could find the method to observe and investigate chemotaxic effect of the substrate into the cell.

For the porous and semi-porous (with the functionalized surface) materials (Fig.9,10) it was observed the surface modification effect into the protein adsorption. For some materials surface functionalization using proteins had a significant effect for the endothelialization process, although that the cracks were visible on the surface. From the material science point of view titanium nitride exhibit the most promising properties. Finally the initial cell density and pore size has a visible effect on full confluence.

For polyelectrolytes, it was observed the unique influence on the frequency after the PEG introduction into the structure. It was postulated that difficulties associated with the efficient PEG incorporation were caused by the diffusion character of the underlying PLL/HA material. We decided to redo the experiment and as a final layer deposit hyaluronic acid. The same material as was deposited before PLL PEG. No frequency change was observed. We rather did not increase the mass of all "layer by layer" structure. Concluding, it is probable that the last layer before PLL PEG diffused into the structure. The difficulties with the PEG 
anchoring came from the fact that in practice we were trying to deposit PLL PEG into PLL which is physically impossible. The literature study is necessary to find the possible explanation.

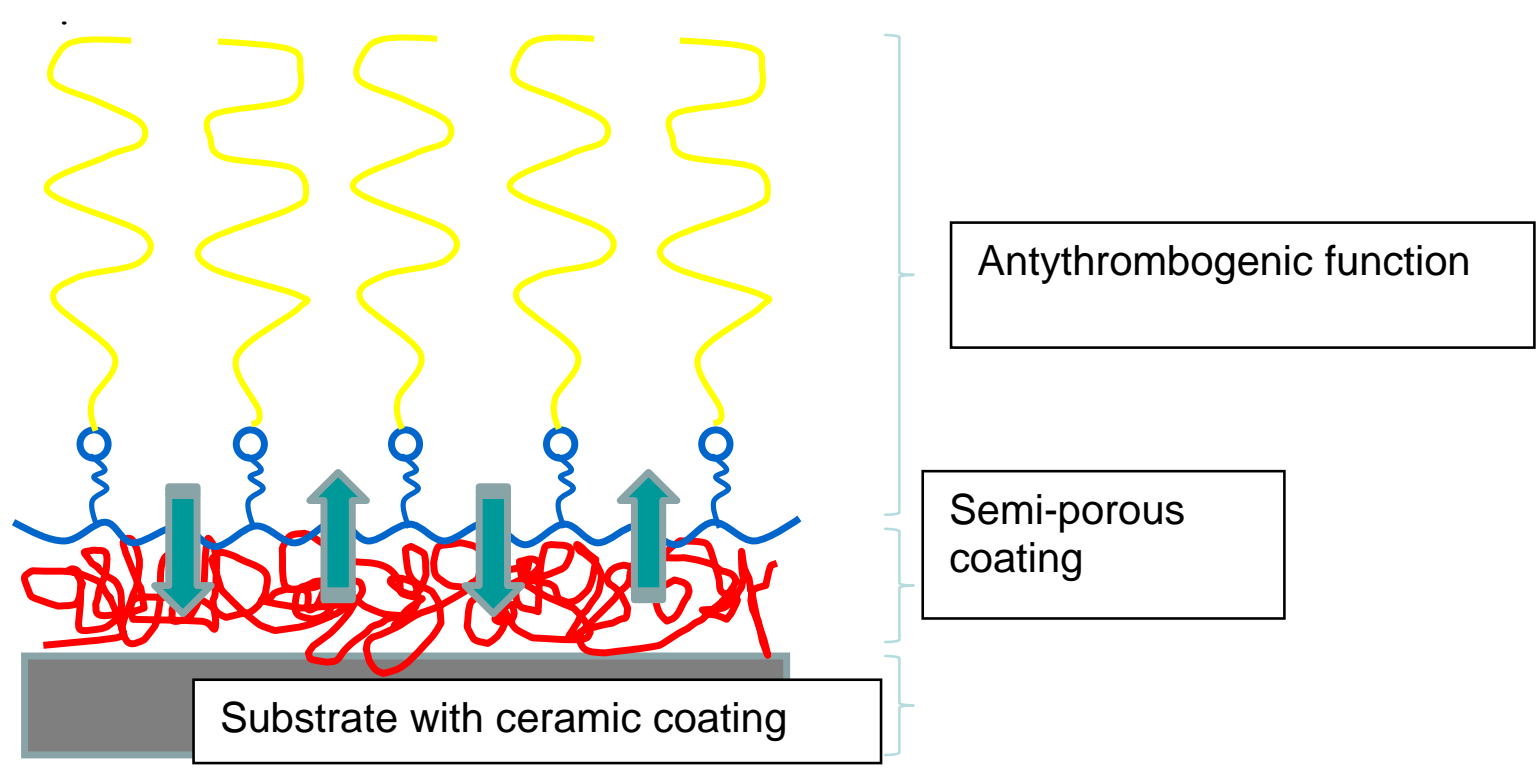

Fig. 10. Architecture of functional semi-porous coating

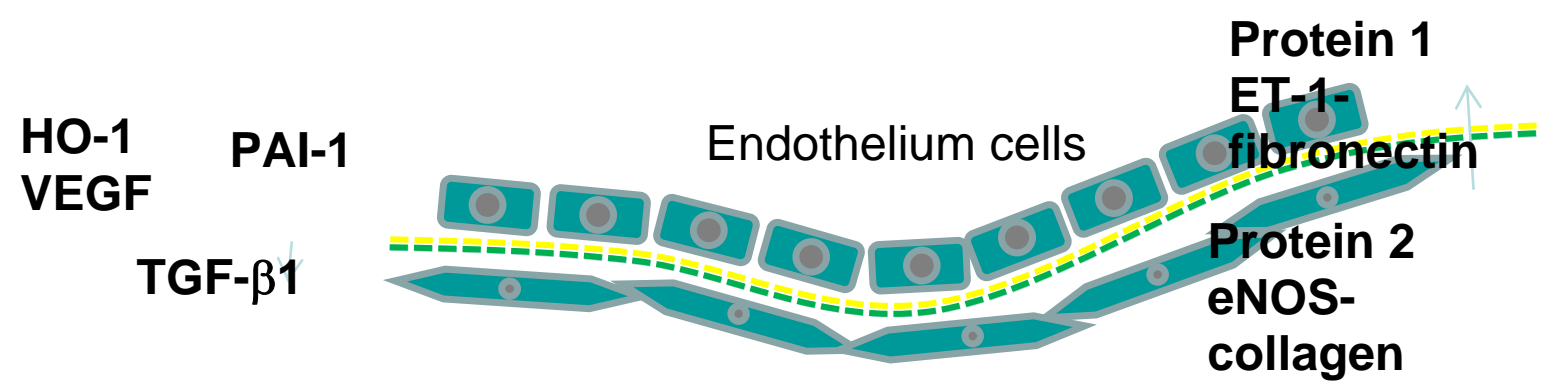

Fig.11. Architecture of functional porous coating

(HO-1 enzyme participating at angiogenesis process; VEGF vascular endothelial growth factor) 
a)

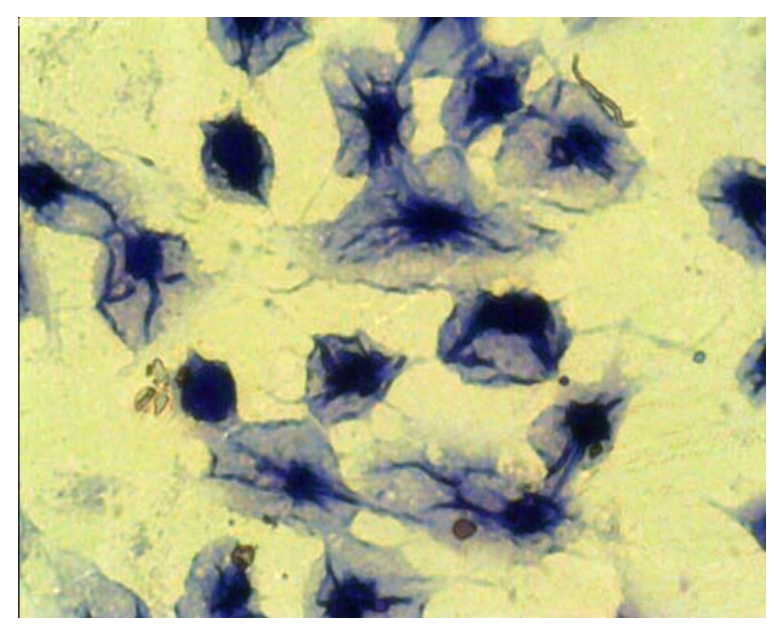

b)

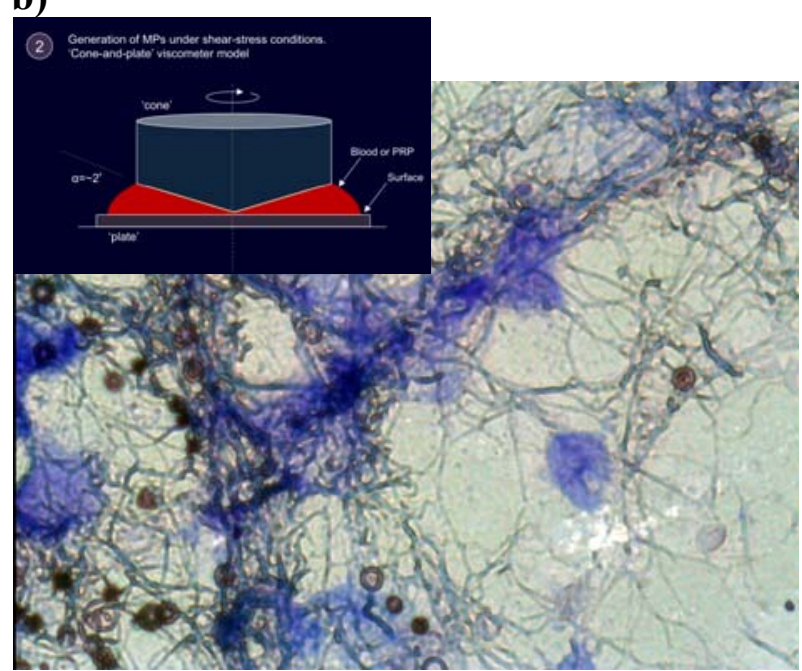

Fig. 12. Blood material interaction; porous coating (PLL+HA)+fibronectin+HUVEC, a) HUVEC cells after deposition, b) HUVEC cells after test impact $R$; possible vascularization

(HUVEC-human umbilical vein endothelial cells; PLL - poly-L-leucine, artificial enzyme; HA- hyaluronic acid)

International cooperation (A, PL, F) realized by the authors between the highly reputable key research groups and industrial partners is of special need in this multidisciplinary activity (materials science - biochemistry - medicine) to achieve an improvement in treatment for patients in the shortest possible time.

\section{CONCLUDING REMARKS}

A development of novel, low thrombogenic materials is of central importance. The presented contribution focused on the processing of the coating with an improved biocompatibility. The microstructure examinations by means of highly specialized methods tested both the quality of the surface morphology from the point of view of its homogeneity and the coating adhesion to the polymer substrate on the cross section.

The intended target of the work was to elaborate materials which would not change the properties of the PU substrate but also effectively separate it from the tissue. The polymer exhibits the change in stiffness in the long term clinical application thus the need of the surface modification. Optimisation of deposition parameter has been established from the point of view of chemical composition and surface morphology as well as satisfactory adhesion. Coatings have been fabricated on thermo-sensitive polymer substrate by application of hybrid method. It was necessary to investigate the thermal effect which could influence in the physical-mechanical parameters changes. It was found that polyurethane is thermally stable. The first effect was observed over 100 deg.C. One can conclude that the process of layer deposition does not influence the polymer degradation. As the argument against is that the additional endothermic effect appeared and the temperature ranges of the glass transformation. This aspect is still under consideration.

A measurement of the strength of the bonds between the biomaterials and the cells is a major challenge in cellular biology, since it allows for the identification of the different species in the adhesion phenomena, thus the cell-material interaction was under examination 
by means of the dynamic test of the cell detachment. The biocompatibility was studied in the static test. As model cells, fibroblasts were taken under consideration. It was observed that it is highly important to focus on the proper phase composition and the stoichiometry of the material coating. The biocompatibility tests revealed the negative effect of the loss of stoichiometry, which was shown especially for titanium nitride.

The cell- material interaction was investigated using cell detachment essay. Cell detachment test gives the overview about the cell behaviour and shear stress which could appear between the cell and the biomaterial. The lowest cell material interaction was found for stoichiometric titanium nitride. There is still opened question which is pointed out by the investigators over the world. Should we deal with low cell material interaction or rather consider their strong influence on the substrate? When the problem concerns the thrombus effects elimination, the answer is simple - no interaction. When one would like to elaborate more sophisticated materials then the other biological phenomena should be analyzed and the answer is not so simple. Biomaterial design requires the biophysical tests as well as biocompatible tests which would illustrate the biological response of the biomaterial into the biological cell. In this case the tests are performed in static conditions. Stoichiometric titanium nitride is known as biocompatible material. A lack of stoichiometry could result in a negative influence on the surrounding tissue which was proved by the fluorescence observations. Cell - material interaction in static conditions revealed stoichiometric titanium nitride as biocompatible material and this behaviour was damage by non-stoichiometry.

Using a simplified experimental setup, based on inexpensive commercial cone-and-plate analyzer, it was managed to reproduce the same events, which seems limiting usage of artificial materials in contact with blood. The advantage of the proposed method is low volume of donor's blood required for testing and higher throughput of the method, enabling parallel testing of materials. This method inherits the same drawbacks, as other experimental setups for hemobiocompatibility testing. Due to a high physiological variation in blood morphology, mainly platelet count, credible results can be obtained only using 8-10 replicates of the test, as was demonstrated by other authors [24,25]. Moreover, the test is also very sensitive to the imperfect smoothness of the surface. This limitation can be evaded using a high quality test plates. Ranking of PU coatings, despite insufficient number of tested plates, revealed that $\operatorname{TiN}, \operatorname{Ti}(\mathrm{C}, \mathrm{N})$ and possibly $\mathrm{Si}+\mathrm{DLC}$ have the best hemobiocompatibility profiles and should be selected for more extensive tests.

For the porous and semi-porous (with the functionalized surface) materials it was observed the surface modification effect into the protein adsorption. For some materials surface functionalization using proteins had a significant effect for the endothelialization process, although that the cracks were visible on the surface. From the material science point of view titanium nitride exhibit the most promising properties. Finally the initial cell density and pore size has a visible effect on full confluence.

\section{ACKNOWLEDGEMENTS}

We acknowledge the financial support by the project CardioBioMat MNT Era-NetMNT/15/2009 Nanostructural materials for implants and cardiovascular biomedical devices and project Polonium "Blood-contacting materials" 22613WE. 


\section{REFERENCES}

1. Ratner B. D, Hoffman A. S, Schoen F. J, Lemons J. E. Biomaterials Science, Copyright Elsevier Inc. 2004.

2. Bozzaro S and Ponte E. Cell adhesion in the life cycle of Dictyostelium, Experientia; 51 (1995) 1175-81.

3. Ashworth J.M and Watts D.J. Metabolism of the cellular slime mould Dictyostelium discoideum grown in axenic culture. Biochem. J. 119(1970)175.

4. Morio T, Urushihara H, Saito T, Ugawa Y, Mizuno H, Yoshida M, Yoshino R, Mitra B.N, Pi M, .Sato T, Takemoto K, Yasukawa H, Williams J, Maeda M. Takeuchi I, Ochiai H and Tanaka Y. The Dictyostelium discoideum developmental cDNA project: generation and analysis of expressed sequence tags from the first-finger stage of development. DNA Res. 5 (1998) 335.

5. E.Decave, Y.Brechet, F.Bruckert, and B.Fourcade, "Peeling model for cell detachment D. Garrivier", Eur. Phys. J., E 8, (2002)79-97.

6. Décavé E, Garrivier D, Bréchet Y, Fourcade B and Bruckert F. Shear Flow-Induced Detachment Kinetics of Dictyostelium discoideum Cells from Solid Substrate Biophysical Journal. 82 (2002) $2383-2395$.

7. Bruckert F, De Cave E, Garrivier D, Cossion P, Brechet Y, Fourcade B and Satre M. Dictyostelium discoideum adhesion and motility under shear flow: experimental and theoretical approaches. Journal of Muscle Research and Cell Motility, Kluwer Academic Publishers, Netherlands. 23(2002) 651-658.

8. Bruckert F, Demily M, Brecht Y, Boulangé L. Kinetics of yeast detachment from controlled stainless steel surfaces; Colloids and Surfaces B, Biointerfaces, Elsevier Inc. 51(2006) 71-79.

9. Bäuerle D. Laser Processing and Chemistry, Springer-Verlag, Berlin, Heidelberg 2000.

10. Lackner J.M, Waldhauser W, Schwarz M, Mahoney L, Major L, Major B. Polymer pre-treatment by linear anode layer source plasma for adhesion improvement of sputtered TiN coatings. Vacuum 83(2009) 302-307.

11. Lackner J.M. Industrially- scaled hybrid Pulsed Laser Deposition at Room Temperature. Published by Orekop sc., Krakow, 2005.

12. Major B, Bruckert F, Lackner J.M, Ebner R, Kustosz R, Lacki P. Coating on TiN and Ti(C,N) basis for biomedical application to blood contact and $\mathrm{TiN} / \mathrm{CrN}$ multilayered tribological systems produced by pulsed laser deposition. Arch.Metal.and Mater. 53 (2008) 39-48.

13. Lackner J.M, Waldhauser W, Alamanou A, Teicher C, Schmied F, Major L, Major B. Mechanisms for self-assembling topography formation in low-temperature vacuum deposition of inorganic coatings on polymer surfaces. Bull.Pol.Ac.:Tech. 58(2) (2010) 281-294.

14. Sarna J, Kustosz R, Major R, Lackner J.M, Major B. Polish Artificial Heart - new coatings, technology, diagnostics. Bull.Pol.Ac.Tech. 58(2) (2010) 329-336.

15. Major R, F.Bruckert F, J.M.Lackner J.M, Waldhauser W, Pietrzyk M, Major B. Kinetics of eucariote cells adhesion under shear flow detachment on the PLD deposited surfaces. Bull.Pol.Ac.Ser.Techn. 56(2008) 223-228.

16. Sanak M, Jakiela B, Wegrzyn W. Assesment of hemocompatibility of materiale with arteria blond flow by platelet functional tests. Bull.Pol.Ac.Tech. 58(2) (2010) 317-322.

17. Thornton J.A, Influence of apparatus geometry and deposition conditions on the structure and topography of thick sputtered coatings. J.Vac.Sci.Technol., 11(4) (1974) 666-670.

18. WinSAM Vario I to III, and SAM 2000, KSI, Kraemer Scientific Instruments, Lerchenweg 1618, 35745 Herborn, Germany. 
19. Ultrasonic testing, J.Krautkraemer, H.Krautkraemer, Springer Verlag, 1986.

20. Acoustic microscopy, Andrew Briggs (Monographs on the physics and chemistry of materials, Vol. 46), Oxford University Press, 1992.

21. EVOLUTION I-III, SAM TEC GmbH, P.O. 3111, 73641 Aalen, Email: info@samtecgermany.com.

22. Seyfert U.T, Biehl V and Schenk J. In vitro hemocompatibility testing of biomaterials according to the ISO 10993-4. Biomol. Eng. 19(2-6) (2002) 91-96.

23. M. Otto M, Klein C.L, Koehler H, Wagner M, Roehrig O and Kirkpatrick C.J. Dymanic blood cell contact with biomaterials: validation of a flow chamber system according to international standards. J Mater Sci Mater Med., 8(3)(1997)119-129.

24. Streller U, Sperling C, Huebner J, Ranke R and Werner C. Design and evaluation of novel blood incubation systems for in vitro hemocompatibility assessment of planar solid surfaces. J. Biomed. Mater. Res. B Appl. Biomater. 66B (1) (2003) 379-390.

25. Schaub R.D, Kameneva M.V, Borovetz H.S and W.R. Wagner W.R. Assessing acute platelet adhesion on opaque metallic and polymeric biomaterials with fiber optic microscopy. J. Biomed. Mater. Res. 49 (4) (2000) 460-468.

26. Sbrana S, Della Pina F, Rizza A, Buffa M, De Filippis R, Gianetti J and Clerico A. Relationships between optical aggregometry (type born) and flow cytometry in evaluating ADP-induced platelet activation. Cytometry B Clin Cytom. 74 (1)(2008)30-39.

27. Nomura S, Shouzu A, Tamoto K, Togane Y, Goto S, Uchiyama S and Ikeda Y. Assessment of an ELISA kit for platelet-derived microparticles by joint research at many institutes in Japan. J. Atheroscler. Thromb. JOI JST.JSTAGE/jat/26432. 2009, published online.

28. Harrison P. Progress in the assessment of platelet function. Br J Hematol. 111(3) (2000) 733-744.

29. Shenkman B., Savion N, Dardik R, Tamarin I and Varon D. Testing of platelet deposition on polystyrene surface under plow conditions by the cone and plate(let) analyser: role of platelet activation, fibrinogen and von Willebrand factor. Thromb. Res. 99 (4) (2000) 353-362.

30. Gemmel C.H. Activation of platelets by in vitro whole blood contact with materials: increases in microparticle procoagulant activity, and soluble P-selectin blood levels. J. Biomater. Sci. Polym. Ed. 12 (8) (2001) 933-934.

31. Varon D, Lashevski I, Brenner B, Beyar R, Lanir N, Tamarin I and Savion N. Cone and plate(let) analyzer: monitoring glycoprotein IIb/IIIa antagonists and von Willebrand disease replacement therapy by testing platelet deposition under flow conditions. Am Heart J. 135 (5 Pt 2 Su)S(1998) 187-193.

32. Nomura S, Ozaki Y and Ikeda Y. Function and role of microparticles in various clinical settings" Thromb. Res. 123 (1) (2008) 8-23.

33. Blann A, Shantsila E and Shantsila A. Microparticles and arterial disease. Semin. Thromb. Hemost. 35 (5) (2009) 488-496.

34. Gemmel C.H, Ramirez S.M, Yeo E.L and Sefton M.V. Platelet activation in whole blood by artificial surfaces: identification of platelet-derived microparticles and activated platelet binding to leukocytes as material-induced activation events. J. Lab. Clin. Med. 125 (2) (1995) 276-287. 